\title{
Arteriosklerose, Restenose und Tumorprogression: PADMA 28 wirkt antiproliferativ auf aktivierte glatte Muskelzellen und Tumorzellen
}

Die Proliferation glatter Muskelzellen der Arterienwand (smooth muscle cells, SMC) spielt bei der Entstehung der Arteriosklerose, einer Restenose nach Angioplastie oder Stenting sowie bei der Tumorprogression eine wichtige Rolle. PADMA 28Extrakt hemmte die Proliferation von unstimulierten sowie von durch die Mitogene bFGF (Basic Fibroblast Growth Factor) und/oder Thrombin-induzierte Proliferation von SMC in vitro. Der antiproliferative Effekt von PADMA 28 zeigte sich auch in aktivierten Tumorzellkulturen und war nach Entfernen des Präparates reversibel, was darauf hindeutet, dass die antiproliferative Wirkung nicht auf einer Zellschädigung beruht. Somit kann PADMA 28 als Anti-Arteriosklerose-Mittel im Sinne einer Prophylaxe eingesetzt werden, um z.B. Intimahyperplasie und postoperative Stenose zu vermeiden; ebenso könnte es in der Vorbeugung und Behandlung anderer Gefässerkrankungen, Tumorwachstum und Metastasierung eine Rolle spielen.

Die Schädigung des Arterienendothels durch arteriosklerotische Veränderungen, aber auch nach operativen Eingriffen wie z.B. perkutaner transluminaler koronarer Angioplastie, Ballondilatation oder Stenting führt zu Gefässverletzungen, die eine Wundheilungsreaktion auslösen. Als Reaktion beginnt die durch Wachstumsfaktoren und Zytokine ausgelöste Proliferation und Migration von SMC der Gefässwand, was unter Umständen zu einer (erneuten) Gefässverengung führen kann. Eingebunden in diesen Prozess sind IL-1 (Interleukin), PDGF (Platelet-derived Growth Factor), bFGF, HB-EGF (Heparin-binding Epidermal Growth Factor) und IGF-I (Insulin-like Growth Factor). Auch Thrombin kann die Proliferation von SMC anregen und zur Intimahyperplasie beitragen.

IGF-I und bFGF spielen auch eine Rolle bei Krebserkrankungen. bFGF, aus der Familie strukturabhängiger kationischer Polypeptide, vermittelt über die Stimulierung von Proliferation und Migration von Endothelzellen, SMC und Fibroblasten die Angioneogenese, welche für die Nährstoffversorgung und Metastasierung von Tumoren wichtig ist.

IGF-I hat verschiedene biologische Funktionen. Neben der insulinähnlichen Wirkung auf Glukoseaufnahme und Glykogensynthese kontrolliert es zusammen mit PDGF und bFGF die Zellproliferation, wirkt mitogen auf SMC und Endothelzellen, fördert die endotheliale Tubulogenese und scheint einen Einfluss auf Zellmotilität und -migration sowie auf die Proteinsynthese zu haben. Ausserdem hemmt es die Apoptose. Der atherogene Effekt von IGF-I wird durch vermehrte IGF-I mRNS in SMC aus arteriosklerotischen Plaques bestätigt. Es ist bekannt, dass es die Aufnahme und Oxidation von LDL-Cholesterin durch Makrophagen fördert.

Ein weiteres Enzym, das die Angioneogenese fördert und damit sowohl bei der Wundheilung als auch bei Tumorerkrankungen eine Rolle spielt, ist die HO-1 (Hämoxygenase). In einer Studie konnte auf Protein- wie auch auf mRNA-Level eine Steigerung der HO-1 in humanen Endothelzellen durch PAMDA 28 gezeigt werden. Dies trägt vermutlich zur antiatherogenen und antientzündlichen Wirkung des Präparats bei, da HO-1 ausserdem auch hemmend auf Entzündungsvorgänge, oxidativen Stress und vermehrte Bindegewebsbildung wirkt.

Solche Wachstumsfaktoren bilden demnach einen gemeinsamen Nenner zwischen Restenose und Tumorprogression, Prozesse, die durch eine überschiessende Zellproliferation, chronisch entzündliche Vorgänge und schlecht kontrollierte
Zellmigration gekennzeichnet sind. Substanzen, die die Effekte von Mitogenen wie IGF-I und bFGF beeinflussen, könnten deshalb in der Behandlung und Prävention von Arteriosklerose, Restenose und Tumorerkrankungen eine Rolle spielen. PADMA 28 (Zulassungsnummer Swissmedic 58436) könnte eine solche Substanz sein. Es ist bekannt für seine anti-oxidative Wirkung, hemmt Proteinasen sowie die Produktion von inflammatorischen Zytokinen in humanen, mononukleären LPS- bzw. LTA-stimulierten Zellen. Ausserdem steigert es die Produktion von HO-1 mit ihrer anti-inflammatorischen und anti-oxidativen Wirkung.

In der vorliegenden Studie wurde die Wirkung von PADMA 28 auf die Proliferation von SMC und Endothelzellen, wie auch der Einfluss des Präparates auf die zelluläre Antwort auf stimulierende Faktoren (Stimulierung von SMC mit bFGF und/oder Thrombin, Stimulierung einer Tumorzell-Linie mit IGF-I), untersucht.

\section{Material und Methoden}

$100 \mathrm{mg}$ PADMA 28 Pulver wurde in $10 \mathrm{ml}$ 0.9\% NaCI-Lösung extrahiert $\left(30 \mathrm{~min}\right.$ bei $37^{\circ} \mathrm{C}$ ) und die unlöslichen Bestandteile abzentrifugiert. SMC wurden aus der Media boviner Aorten isoliert. Nach 48 h in Kultur wurden sie in Anwesenheit verschiedener Konzentrationen von PADMA 28-Extrakt mit Wachstumsfaktoren (bFGF, Thrombin) stimuliert.

Zellproliferationsraten wurden durch Zellzählung, die DNA-Syntheserate über die Inkorporation von radioaktivem $\left({ }^{3} \mathrm{H}\right)$ Thymidin oder die Absorptionsrate bei der Wellenlänge $550 \mathrm{~nm}$ gemessen. Die Experimente wurden je dreifach durchgeführt, die Streuung überstieg nicht $\pm 20 \%$.

\section{Resultate}

PADMA 28-Extrakt zeigte einen dosisabhängigen antiproliferativen Effekt an unstimulierten SMC, wobei die Proliferation bei $40 \mu \mathrm{g} / \mathrm{ml} \mathrm{um} 40 \%$ und bei $60 \mu \mathrm{g} / \mathrm{ml} \mathrm{um} 80 \%$ gehemmt wurde (Abb. 1). Im Gegensatz dazu wurde das Wachstum von Endothelzellen kaum gehemmt. Die antiproliferative Wirkung von PADMA 28 zeigte sich auch in einer verminderten DNA-Syntheserate in durch Thrombin und/ oder bFGF stimulierten SMC. Thrombin $(7 \mathrm{nM})$ steigerte die DNA-Synthese um das 3-Fache, bFGF (1ng/ml) um das 5Fache und die Kombination um das 16-Fache. PADMA 28Extrakt $(60 \mu \mathrm{g} / \mathrm{ml})$ blockierte diese Aktivierung durch Thrombin und bFGF und verhinderte auch den stark syn- 


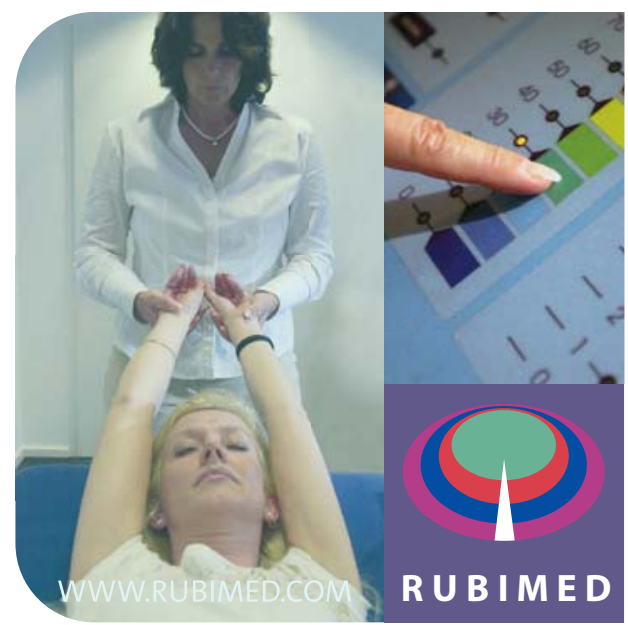

Psychosomatische Energetik

- Neuartiges System der Energiemedizin: hocheffektiv, preiswert

- erstmals präzise Testung des Energiefeldes („Aura-Blutdruck“)

- Erkennen der wahren Krankheitsursachen und „Energieräuber“

- Sanfte spirituelle Reifung und ganzheitliche Heilung

- „Psychotherapie mit homöopathischen Tropfen“

Für jeden der mehr über sich und seinen unbewußten Charakter erfahren möchte. Rubimed AG, Grossmatt 3, 6052 Hergiswil, Tel. 041-630 0888 www.rubimed.com, Email info@rubimed.com

\section{Die einzigartige Wirkungsweise}

\section{Spenglersan Nasenspray gegen Schnupfen}

Mit Spenglersan Virus influencae comp. D9-G Nasenspray steht ein Schnupfenmittel zur Verfügung, das weit mehr bewirkt, als nur die Schleimhäute abzuschwellen.

\section{Spenglersan - einfach sanft!}




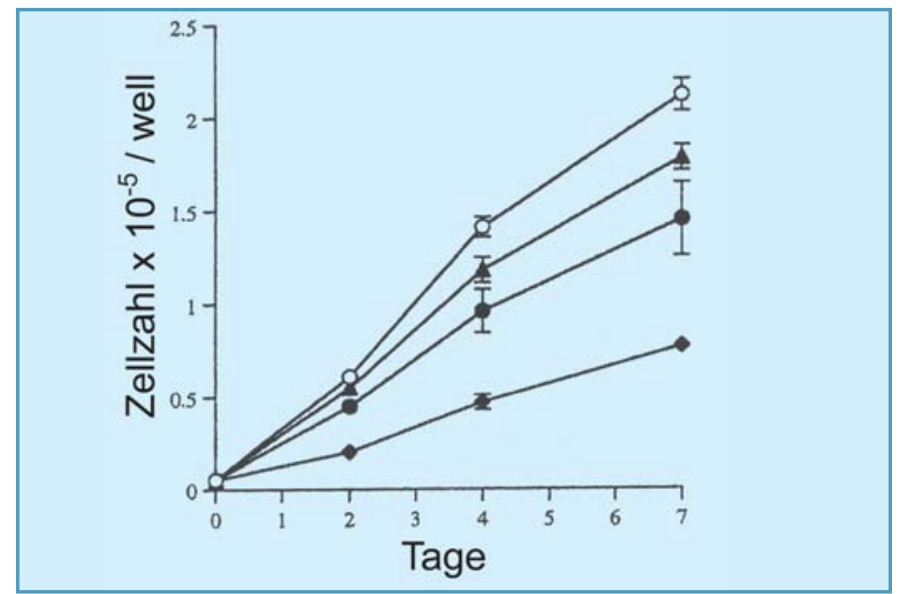

Abb. 1. Wässriger PADMA 28-Extrakt hemmte die Proliferation vaskulärer SMC dosisabhängig (o Control; $\Delta 20 \mu \mathrm{g} / \mathrm{ml}$; $40 \mu \mathrm{g} / \mathrm{ml} ; \quad 60 \mu \mathrm{g} / \mathrm{ml}$ ).

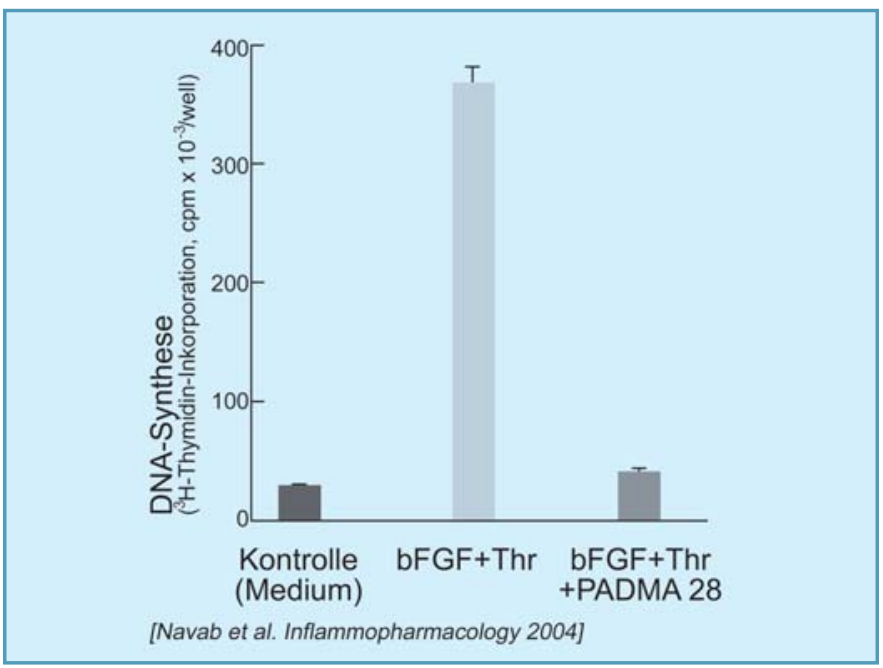

Abb. 2. Proliferation von vaskulären glatten Muskelzellen (SMC), dargestellt anhand des Einbaus von ${ }^{3} \mathrm{H}$-Thymidin in neu synthetisierte DNA. Die DNA-Syntheserate war im Gegensatz zu unstimulierten SMC (PBS) in mit basic Fibroblast Growth Factor (bFGF) und Thrombin (Thr)-stimulierten SMC stark erhöht. Die Inkubation mit PADMA 28-Extrakt $(60 \mu \mathrm{g} /$ $\mathrm{ml}$ ) blockierte diese Aktivierung und verhinderte insbesondere auch den starken synergistischen Effekt der beiden Wachstumsfaktoren.

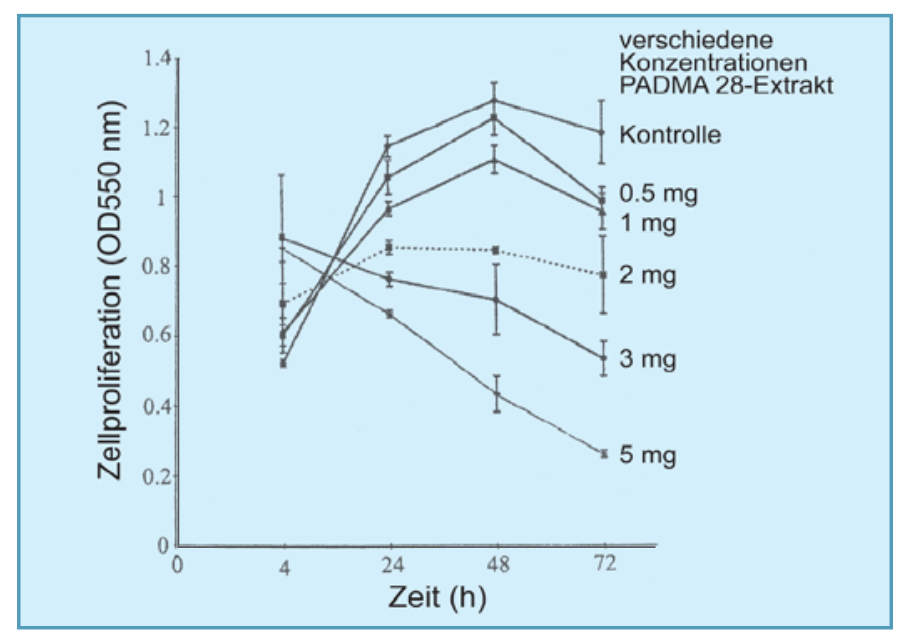

Abb. 3. Hemmung der proliferativen Wirkung von IGF-I auf H-59 Tumorzellen durch PADMA 28. $1 \mathrm{mg} / \mathrm{ml}$ reduzierte die IGF-l-induzierte DNASyntehese um 35\%, 2 mg/ml blockierte sie fast vollständig. ergistischen Effekt der beiden Wachstumsfaktoren (Abb. 2). Die Möglichkeit, dass PADMA 28 auf Grund der enthaltenen Polyanionen den kationischen bFGF unspezifisch hemmte, wurde ausgeschlossen, da die stark anionischen L-Asparagin- und L-Glutaminsäuren keine Hemmung bewirkten.

Der Einfluss von PADMA 28 auf die mitogene Wirkung von IGF-I wurde an H-59 Tumorzellen untersucht, da diese Zelllinie funktionale IGF-I-Rezeptoren exprimiert und zuverlässige, reproduzierbare Reaktionen auf IGF-I zeigt. PADMA $28(1 \mathrm{mg} / \mathrm{ml})$ reduzierte die IGF-I-induzierte Proliferation in den Zellen um 35\% bzw. blockiert sie vollständig (2mg/ml) (Abb. 3).

\section{Diskussion}

Verschiedene Wachstumsfaktoren und Zytokine wie z.B. IL1, PDGF, bFGF, IGF-I und Thrombin sind verantwortlich für die Proliferation und Migration von SMC, welche in arteriosklerotischen Prozessen, aber auch im Krebsgeschehen eine Rolle spielen. Medikamente, welche die proliferativen Effekte von einem oder mehreren dieser Faktoren hemmen, könnten in der Behandlung und Prävention solcher Erkrankungen eingesetzt werden.

Die vorliegenden Resultate zeigen eine starke antiproliferative Wirkung von PADMA 28 auf unstimulierte und stimulierte SMC, Endothelzellen werden kaum beeinflusst. Diese selektive Hemmung schliesst aus, dass die Wachstumshemmung der SMC durch einen trivialen zytotoxischen Wirkmechanismus erzielt wird. Intakte Endothelzellen sind insbesondere wichtig, denn nur mit einer funktionierenden Abdeckung ist die atherosklerotische Plaque stabil zu halten. Das Präparat hemmt ausserdem die Proliferation von IGF-I-stimulierten H-59 Tumorzellen und von bFGF- und Thrombin-stimulierten SMC. Hier durchbricht es den starken Synergismus zwischen den beiden Faktoren. Eine reine elektrostatische Neutralisierung dieser kationischen PeptidWachstumsfaktoren konnte ausgeschlossen werden.

PADMA 28 kann auch auf einen weiteren Prozess bei der Atherogenese einwirken. Die Hemmung der IGF-I-Signalweiterleitung kann die Entwicklung arteriosklerotischer Läsionen begrenzen und die Intimahyperplasie und somit die Stenosierung verhindern. Die Hemmung der Proliferation sowohl von unstimulierten als auch von stimulierten SMC weist auf den möglichen therapeutischen Nutzen des Pflanzenpräparates in der Co-Medikation nach Koronararterien-Interventionen hin. Auch bei der Behandlung von arteriosklerosebedingten Durchblutungsstörungen verspricht das Tibetische Vielstoffgemisch neben der Eindämmung der entzündlichen Vorgänge eine drastische Verlangsamung des Stenosierungsprozesses.

Barak V, Kalickman I, Halperin T et al.: Padma 28, A Tibetan herbal preparation is an inhibitor of inflammatory cytokine production. Eur Cytokine Netw 2004;15(3):203-9. Exner M, Raith M, Holzer G et al.: Anti-inflammatory mechanisms of the Tibetan herbal preparation Padma 28 in the vessel wall. Forsch Komplementärmed 2006; 13(Suppl 1):13-7.

Ginsburg I, Sadovnik M, Sallon S et al.: Padma 28, a traditional Tibetan herbal preparation inhibits the respiratory burst in human neutrophils, the killing of epithelial cells by mixtures of oxidants and pro- inflammatory agonists and peroxidation of lipids. Inflammopharmacology 1999;7(1):47-62.

Navab R et al.: Padma 28, a traditional Tibetan herbal preparation, blocks cellular responses to bFGF and IGF-1. Inflammopharmacology 2004;12(4):373-89. 